\title{
New Periodic Solutions for a Class of Zakharov Equations
}

\author{
Cong Sun ${ }^{1,2}$ and Shuguan Ji ${ }^{1}$ \\ ${ }^{1}$ College of Mathematics, Jilin University, Changchun 130012, China \\ ${ }^{2}$ The City College of Jilin Jianzhu University, Changchun 130111, China
}

Correspondence should be addressed to Shuguan Ji; jisg@jlu.edu.cn

Received 10 May 2016; Revised 15 August 2016; Accepted 1 September 2016

Academic Editor: Mariano Torrisi

Copyright (C) 2016 C. Sun and S. Ji. This is an open access article distributed under the Creative Commons Attribution License, which permits unrestricted use, distribution, and reproduction in any medium, provided the original work is properly cited.

Through applying the Jacobian elliptic function method, we obtain the periodic solution for a series of nonlinear Zakharov equations, which contain Klein-Gordon Zakharov equations, Zakharov equations, and Zakharov-Rubenchik equations.

\section{Introduction}

For most of nonlinear evolution equations, we have many methods to obtain their exactly solutions, such as hyperbolic function expansion method [1], the transformation method [2], the trial function method [3], the automated method [4], and the extended tanh-function method [5]. But these methods can only obtain solitary wave solution and cannot be used to deduce periodic solutions. The Jacobian function method provides a way to find periodic solutions for some nonlinear evolution equations. In particular, in the research of plasma physics theory, quantum mechanics, fluid mechanics, and optical fiber communication, we frequently meet kinds of Zakharov equations.

Hence, in this paper, inspired by Angulo Pava's work [6], we are concerned to obtain exact periodic solutions of a series of Zakharov equations,

$$
\begin{aligned}
u_{t t}-u_{x x}+u+n u & =0, \\
n_{t t}-n_{x x} & =\left(|u|^{2}\right)_{x x}, \\
u_{t t}-u_{x x}-|v|_{x x} & =0 \\
i v_{t}+\alpha v_{x x}-u v & =0,
\end{aligned}
$$

and Zakharov-Rubenchik equations,

$$
\begin{aligned}
i B_{t}+\omega B_{x x}-k\left(u-\frac{1}{2} \lambda \rho+q|B|^{2} B\right) & =0, \\
\theta \rho_{t}+(u-\lambda \rho)_{x} & =-k|B|_{x}^{2}, \\
\theta u_{t}+(\beta \rho-\lambda u)_{x} & =\frac{1}{2} k \lambda|B|_{x}^{2} .
\end{aligned}
$$

\section{The Periodic Solution for Klein-Gordon Zakharov Equations}

The Klein-Gordon Zakharov equations are used to show the interaction between langmuir wave and ion wave in the plasma, which has the following form:

$$
\begin{aligned}
u_{t t}-u_{x x}+u+n u & =0, \\
n_{t t}-n_{x x} & =\left(|u|^{2}\right)_{x x},
\end{aligned}
$$

where $u(x, t)$ denotes the biggest moment scale component produced by electron in electric field. $n(x, t)$ denotes the speed of deviations between the ions at any position and that at equilibrium position.

Now, we suppose that it possesses solitary wave solutions of the following form:

$$
\begin{aligned}
u(x, t) & =e^{i \theta} \varphi(\xi), \\
n(x, t) & =n(\xi), \\
\xi & =x-c t,
\end{aligned}
$$

where $c$ is a traveling wave speed and $c^{2}<1$ is a constant.

By substituting (4) into (3), we can obtain

$$
\begin{aligned}
c^{2} \varphi^{\prime \prime}-\varphi^{\prime \prime}+\varphi+\varphi n & =0, \\
\left(c^{2}-1\right) n^{\prime \prime} & =\left(\varphi^{2}\right)^{\prime \prime} .
\end{aligned}
$$


By (6), we have

$$
\left(c^{2}-1\right) n-\varphi^{2}=c_{1} \xi+m,
$$

where $c_{1}, m$ are integration constants.

In what follows, we are concerned with the periodic solution of (3); thus we need to require $c_{1}=0$.

Therefore, (7) implies

$$
n=\frac{\varphi^{2}+m}{c^{2}-1}
$$

Moreover, through (8) and (5), we have that

$$
\left(c^{2}-1\right) \varphi^{\prime \prime}+\varphi+\frac{\varphi^{3}+\varphi m}{c^{2}-1}=0 .
$$

Multiplying (9) by $\varphi^{\prime}$ and integrating once, we obtain

$$
\frac{c^{2}-1}{2}\left(\varphi^{\prime}\right)^{2}+\frac{\varphi^{2}}{2}+\frac{\varphi^{4}+2 m \varphi^{2}}{c^{2}-1} \frac{1}{4}=h,
$$

where $h$ is a nonzero integration constant. Furthermore, $h, m$, and $c$ satisfy the following condition:

$$
\begin{aligned}
& \left(1-c^{2}-m\right)^{2}-4 h\left(1-c^{2}\right) \geq 0 \\
& \left(1-c^{2}-m\right)-\sqrt{\left(1-c^{2}-m\right)^{2}-4\left(1-c^{2}\right) h} \geq 0, \\
& 0<h<\frac{\left(1-c^{2}-m\right)^{2}}{4\left(1-c^{2}\right)}
\end{aligned}
$$

so that

$$
\left(\varphi^{\prime}\right)^{2}=\frac{1}{2} \frac{1}{\left(c^{2}-1\right)^{2}}\left(\eta_{1}^{2}-\varphi^{2}\right)\left(\varphi^{2}-\eta_{2}^{2}\right),
$$

where $-\eta_{1}, \eta_{1},-\eta_{2}$, and $\eta_{2}$ are the zeros of the polynomial $F(\lambda)=-\left(\left(c^{2}-1+m\right) /\left(c^{2}-1\right)^{2}\right) \lambda^{2}-(1 / 2)\left(\lambda^{4} /\left(c^{2}-1\right)^{2}\right)+2 h /\left(c^{2}-\right.$ $1)$. Without losing generality, we assume that $\eta_{1}>\eta_{2}>0$. Therefore, we can deduce that $\eta_{1} \leq \varphi \leq \eta_{2} ; \eta_{1}$ and $\eta_{2}$ satisfy

$$
\begin{aligned}
\eta_{1}^{2}+\eta_{2}^{2} & =2\left(1-c^{2}\right)-2 m, \\
\eta_{1}^{2} \eta_{2}^{2} & =4 h\left(1-c^{2}\right)
\end{aligned}
$$

as

Let $\phi=\varphi / \eta_{1}, k^{2}=\left(\eta_{1}^{2}-\eta_{2}^{2}\right) / \eta_{1}^{2}$. Hence, (12) can be written

$$
\left(\phi^{\prime}\right)^{2}=\frac{1}{2} \frac{1}{\left(c^{2}-1\right)^{2}} \eta_{1}^{2}\left(1-\phi^{2}\right)\left(\phi^{2}-1+k^{2}\right) .
$$

Moreover, we define a new variable $\psi$, which satisfies $\psi^{\prime} \geq$ $0, \psi(0)=0$, by the relation $\phi^{2}=1-k^{2} \sin ^{2} \psi$; through a tedious computation, we obtain that

$$
\left(\psi^{\prime}\right)^{2}=\frac{1}{2} \frac{1}{\left(c^{2}-1\right)^{2}} \eta_{1}^{2}\left(1-k^{2} \sin ^{2} \psi\right) .
$$

Then we obtain

$$
\int_{0}^{\psi(\xi)} \frac{d \tau}{\sqrt{1-k^{2} \sin ^{2} \tau}}=\frac{\sqrt{2}}{2} \frac{1}{1-c^{2}} \eta_{1} \xi .
$$

According to the definition of the Jacobian elliptic function $y=\operatorname{sn}(u ; k)$, we can obtain that $\sin \psi=\operatorname{sn}\left(\eta_{1} \sqrt{\beta} \xi, k\right)$. Here, $\beta=\sqrt{2} / 2\left(1-c^{2}\right)$. So

$$
\begin{aligned}
\phi(\xi) & =\sqrt{1-k^{2} \operatorname{sn}^{2}\left(\eta_{1} \frac{\sqrt{2}}{2} \frac{1}{1-c^{2}} \xi, k\right)} \\
& =\operatorname{dn}\left(\eta_{1} \frac{\sqrt{2}}{2} \frac{1}{1-c^{2}} \xi, k\right) .
\end{aligned}
$$

By returning to initial variable, we obtain that

$$
\varphi(\xi)=\eta_{1} \operatorname{dn}\left(\eta_{1} \frac{\sqrt{2}}{2} \frac{1}{1-c^{2}} \xi, k\right)
$$

is a dnoidal solution of (10).

Furthermore, dn has fundamental period $2 K(k)$; that is, $\operatorname{dn}(u+2 k ; k)=\operatorname{dn}(u ; k)$, and $K(k)$ is the complete elliptic integral of first kind. So the dnoidal wave solution $\varphi$ has fundamental period, $T$, given by

$$
T=\frac{2 \sqrt{2} K(k)\left(1-c^{2}\right)}{\eta_{1}} .
$$

So, by applying the method of the Jacobian elliptic function and inspired by Angulo Pava's ideas, we obtain that (3) has the periodic traveling solution of the following form:

$$
\begin{aligned}
& u(x, t)=e^{i \theta} \varphi(\xi)=e^{i \theta} \eta_{1} \operatorname{dn}\left(\eta_{1} \frac{\sqrt{2}}{2} \frac{1}{1-c^{2}} \xi, k\right), \\
& n(x, t) \\
& =\frac{\left(\eta_{1} \operatorname{dn}\left(\eta_{1}(\sqrt{2} / 2)\left(1 /\left(1-c^{2}\right)\right) \xi, k\right)\right)^{2}+m}{c^{2}-1} .
\end{aligned}
$$
form:

Moreover, $T$ and $k^{2}$ can be also rewritten as the following

$$
\begin{aligned}
T\left(\eta_{2}\right) & =\frac{2 \sqrt{2} K\left(k\left(\eta_{2}\right)\right)\left(1-c^{2}\right)}{\sqrt{2\left(1-c^{2}\right)-2 m-\eta_{2}^{2}}}, \\
k^{2} & =\frac{2\left(1-c^{2}\right)-2 m-2 \eta_{2}^{2}}{2\left(1-c^{2}\right)-2 m-\eta_{2}^{2}} .
\end{aligned}
$$

Furthermore, if $\eta_{2} \rightarrow 0$ then $k\left(\eta_{2}\right) \rightarrow 1$. Hence $K\left(k\left(\eta_{2}\right)\right) \rightarrow+\infty$, so that $T\left(\eta_{2}\right) \rightarrow+\infty$. If $\eta_{2} \rightarrow \sqrt{1-c^{2}-m}$, then $k\left(\eta_{2}\right) \rightarrow 0$. Hence $K\left(k\left(\eta_{2}\right)\right) \rightarrow \pi / 2$, so that $T\left(\eta_{2}\right) \rightarrow$ $\sqrt{2} \pi\left(1-c^{2}\right) / \sqrt{1-c^{2}-m}$.

Next we will show that, for an arbitrary but fixed $L, \sqrt{1-\left(L^{2}+L \sqrt{L^{2}-8 \pi m}\right) / 4 \pi}<\left|c_{0}\right|<$ $\sqrt{1+\left(L^{2}+L \sqrt{L^{2}-8 \pi m}\right) / 4 \pi}$. We can obtain that there 
is a unique $\eta_{2,0}=\eta_{2,0}\left(c_{0}\right) \in\left(0, \sqrt{1-c_{0}^{2}-m}\right)$ such that $T\left(\eta_{2}\right)=T\left(\eta_{2}(c)\right)=L$ is a fundamental period for the dnoidal wave solution (18).

Theorem 1. Let $L>0$ be arbitrary but fixed. Consider $\sqrt{1-\left(L^{2}+L \sqrt{L^{2}-8 \pi m}\right) / 4 \pi}<\left|c_{0}\right|<$ $\sqrt{1+\left(L^{2}+L \sqrt{L^{2}-8 \pi m}\right) / 4 \pi}$ and unique $\eta_{2,0}=\eta_{2,0}\left(c_{0}\right) \epsilon$ $\left(0, \sqrt{1-c_{0}^{2}-m}\right)$, such that $T\left(\eta_{2}\right)=L$. Then, there exist an interval $U\left(c_{0}\right)$, an interval $I\left(\eta_{2,0}\right)$, and a unique smooth function $F: U\left(c_{0}\right) \rightarrow I\left(\eta_{2,0}\right)$ such that $F(c)=\eta_{2}$ and

$$
L=\frac{2 \sqrt{2} K\left(k\left(\eta_{2}\right)\right)\left(1-c^{2}\right)}{\sqrt{2\left(1-c^{2}\right)-2 m-\eta_{2}^{2}}},
$$

where $c \in U\left(c_{0}\right), \eta_{2} \in U\left(\eta_{2,0}\right)$.

Proof. Based on the ideas establish in Angulo Pava's work [6], we will give a brief proof. Now, we consider the open set

$$
\begin{aligned}
\Omega & =\left\{\left(\eta_{2}, c\right): \eta_{2} \in\left(0, \sqrt{1-c_{0}^{2}-m}\right), c\right. \\
& \in\left(-\sqrt{1+\frac{L^{2}+L \sqrt{L^{2}-8 \pi m}}{4 \pi}},\right. \\
& \left.\sqrt{1+\frac{L^{2}+L \sqrt{L^{2}-8 \pi m}}{4 \pi}}\right) \cup(-\infty, \\
& \left.-\sqrt{1-\frac{L^{2}+L \sqrt{L^{2}-8 \pi m}}{4 \pi}}\right) \\
& \left.\cup\left(\sqrt{1-\frac{L^{2}+L \sqrt{L^{2}-8 \pi m}}{4 \pi}},+\infty\right)\right\} \in R^{2} .
\end{aligned}
$$

We define $\Phi: \Omega \rightarrow R$ by

$$
\Phi\left(\eta_{2}, c\right)=\frac{2 \sqrt{2} K\left(k\left(\eta_{2}\right)\right)\left(1-c^{2}\right)}{\sqrt{2\left(1-c^{2}\right)-2 m-\eta_{2}^{2}}} .
$$

Here, $k^{2}=\left(2\left(1-c^{2}\right)-2 m-2 \eta_{2}^{2}\right) /\left(2\left(1-c^{2}\right)-2 m-\eta_{2}^{2}\right)$. Hypothesise $\Phi\left(\eta_{2,0}, c_{0}\right)=L$. In what follows, we will prove that $\partial \Phi / \partial \eta_{2}<0$.

From (24), we can obtain that

$$
\begin{aligned}
\frac{\partial \Phi}{\partial \eta_{2}}= & \frac{2 \sqrt{2} \eta_{2} K\left(k\left(\eta_{2}\right)\right)\left(1-c^{2}\right)}{\left(2\left(1-c^{2}\right)-2 m-\eta_{2}^{2}\right)^{3 / 2}} \\
& +\frac{2 \sqrt{2}\left(1-c^{2}\right)}{\sqrt{2\left(1-c^{2}\right)-2 m-\eta_{2}^{2}}} \frac{d K}{d k} \frac{\partial k}{\partial \eta_{2}} .
\end{aligned}
$$

From $k^{2}$, we can deduce $k\left(\eta_{2}, c\right)$ is a strictly decreasing function of $\eta_{2}$.

According to Jacobian elliptic function theory [7], we have

$$
\begin{aligned}
& \frac{d K}{d k}=\frac{E-\left(1-k^{2}\right) K}{k\left(1-k^{2}\right)}, \\
& \frac{d E}{d k}=\frac{E-K}{k} .
\end{aligned}
$$

Here, $E$ is the complete elliptic integral of second kind.

Next, we adopt the reduction to absurdity to prove $\partial \Phi / \partial \eta_{2}<0$. Now, we assume that $\partial \Phi / \partial \eta_{2} \geq 0$. So, we have the following inequality:

$$
\begin{aligned}
& k\left(2\left(1-c^{2}-m\right)-\eta_{2}^{2}\right) K\left(k\left(\eta_{2}\right)\right) \\
& \quad \geq 2\left(1-c^{2}-m\right) \frac{d K}{d k} .
\end{aligned}
$$

Indeed, substituting (26) into (25) and using the method of enlarging and reducing, we obtain that

$$
\begin{aligned}
& \left(1-k^{2}\right)\left[4\left(1-c^{2}-m\right)-2 \eta_{2}^{2}\right] K(k) \\
& \geq 2\left(1-c^{2}-m\right) E(k) .
\end{aligned}
$$

From $k^{2}$, we can easily deduce that

$$
\begin{aligned}
1-k^{2} & =\frac{\eta_{2}^{2}}{2\left(1-c^{2}\right)-2 m-\eta_{2}^{2}}, \\
2-k^{2} & =\frac{2\left(1-c^{2}\right)-2 m}{2\left(1-c^{2}\right)-2 m-\eta_{2}^{2}} .
\end{aligned}
$$

Hence,

$$
2\left(1-k^{2}\right) K(k) \geq\left(2-k^{2}\right) E(k) .
$$

Let $\gamma^{2}\left(\eta_{2}, c\right)=1-k^{2}, \gamma^{\prime}=-k\left(\partial k / \partial \eta_{2}\right)(1 / \gamma)>0$. So, $\gamma$ is an increasing function of $\eta_{2} \in\left(0, \sqrt{1-c^{2}-m}\right)$. Moreover, $\gamma(0)=1, \gamma\left(\sqrt{1-c^{2}-m}\right)=1$.

Define

$$
f(\gamma)=\left(1+\gamma^{2}\right) E\left(\sqrt{1-\gamma^{2}}\right)-2 \gamma^{2} K\left(\sqrt{1-\gamma^{2}}\right) .
$$

Due to $\partial \Phi / \partial \eta_{2} \geq 0, f(\gamma) \leq 0$.

However, by (27) and a simple computation,

$$
f^{\prime}(\gamma)=3 \gamma\left(E\left(\sqrt{1-\gamma^{2}}\right)-K\left(\sqrt{1-\gamma^{2}}\right)\right)<0,
$$

so $f(\gamma)$ is a decreasing function. Furthermore, $f(1)=0$, so for $\gamma \in(0,1), f(\gamma)>0$.

It is in conflict with our assumption. So we obtain our affirmation that $\partial \Phi / \partial \eta_{2}<0$. Hence, by the implicit function theorem, there exists a unique smooth function $F$, defined in a neighborhood, $U\left(c_{0}\right)$ of $c_{0}$, so that $\Phi\left(\eta_{2}(c), c\right)=L$ for $c \in U\left(c_{0}\right)$. Hence, we obtain (22). 


\section{The Periodic Solution for Zakharov Equations}

Now, we consider the following Zakharov equations:

$$
\begin{gathered}
u_{t t}-u_{x x}-(|v|)_{x x}^{2}=0, \\
i v_{t}-\alpha v_{x x}-u v=0,
\end{gathered}
$$

which describe the high frequency moment of plasma, where $u(x, t)$ denotes the ion number density variation, $v(x, t)$ denotes the electric field intensity of slowly varying amplitude, and $\alpha \in R$.

We seek the solitary solutions of (34) in the form

$$
\begin{aligned}
& u=u(\xi), \\
& v=\varphi(\xi) e^{i \xi}, \\
& \xi=x-c t,
\end{aligned}
$$

where $u$ and $\varphi$ are real functions, $c$ is a traveling speed, and $c^{2} \neq 1$.

Substituting (35) into (34), we can obtain that

$$
\begin{aligned}
\left(c^{2}-1\right) u^{\prime \prime}-\left(\varphi^{2}\right)^{\prime \prime} & =0, \\
-\alpha \varphi^{\prime \prime}-(c+2 \alpha) i \varphi^{\prime}+(c+\alpha) \varphi-u \varphi & =0 .
\end{aligned}
$$

By (36), we can deduce

$$
\left(c^{2}-1\right) u-\varphi^{2}=c_{2} \xi+r .
$$

Here, $c_{2}, r$ are integration constants.

Next, we consider periodic solutions of (34). So, we need to require $c_{2}=0$. Therefore, by (38)

$$
u=\frac{\varphi^{2}+r}{c^{2}-1} .
$$

Furthermore, by (37), we can obtain that

$$
c=-2 \alpha \text {. }
$$

So, by (39) and (40), (37) can be rewritten:

$$
\varphi^{\prime \prime}+\varphi\left(1+\frac{2 r}{c-c^{3}}\right)+\frac{2 \varphi^{3}}{c-c^{3}}=0 .
$$

Multiplying (41) by $\varphi^{\prime}$ and integrating once, we obtain

$$
\frac{1}{2}\left(\varphi^{\prime}\right)^{2}+\frac{1}{2} \varphi^{2}\left(1+\frac{2 r}{c-c^{3}}\right)+\frac{1}{2} \varphi^{4}=h,
$$

where $h$ is a nonzero integration constant.

Hence,

$$
\left(\varphi^{\prime}\right)^{2}=\left(a^{2}+\varphi^{2}\right)\left(b^{2}-\varphi^{2}\right) \text {. }
$$

Here, $-a i, a i,-b$, and $b$ are polynomial roots of $F(t)=-t^{4}-$ $t^{2}\left(a^{2}-b^{2}\right)+2 h, b>0$, and

$$
\begin{aligned}
a^{2}-b^{2} & =1+\frac{2 r}{c-c^{3}}, \\
a^{2} b^{2} & =2 h .
\end{aligned}
$$

Let $\chi=\varphi / b$ (suppose $\chi(0)=0)$ and $k^{2}=b^{2} /\left(a^{2}+b^{2}\right)$. Hence, (43) becomes

$$
\left(\chi^{\prime}\right)^{2}=b^{2}\left(1-\chi^{2}\right)\left(\frac{a^{2}}{b^{2}}+\chi^{2}\right) .
$$

Now, we define $\chi^{2}=1-\sin ^{2} \psi$, so we get that

$$
\left(\psi^{\prime}\right)^{2}=\left(a^{2}+b^{2}\right)\left(1-k^{2} \sin ^{2} \psi\right) .
$$

Let $\tau=\sqrt{a^{2}+b^{2}}$. According to the definition of the Jacobian elliptic function sn, we can obtain $\sin \psi(\xi)=$ $\operatorname{sn}(\tau \xi, k)$. So that, $\chi^{2}=1-\operatorname{sn}^{2}(\tau \xi, k)$.

So, we obtain the solution of (41):

$$
\varphi(\xi)=b \operatorname{cn}(\tau \xi, k) .
$$

According to Jacobian elliptic functions theory, $\mathrm{cn}$ has period $4 K(k)$, and we can obtain that the cnoidal wave solution has period $T$, which is given by

$$
T=\frac{4 K(k)}{\tau} \text {. }
$$

So, we can obtain the periodic solutions of (34):

$$
\begin{aligned}
& u(x, t)=u(\xi)=\frac{(b \mathrm{cn}(\tau \xi, k))^{2}+r}{c^{2}-1}, \\
& v(x, t)=v(\xi)=b e^{i \xi} \mathrm{cn}(\tau \xi, k) .
\end{aligned}
$$

Furthermore, it follows that for $k^{2}=b^{2} /\left(a^{2}+b^{2}\right)$, we have $k^{2} \in(0,1 / 2)$.

If $\tau \rightarrow 0$, then $k \rightarrow 0$, and $K(k) \rightarrow \pi / 2, T(\tau) \rightarrow+\infty$. Furthermore, if $\tau \rightarrow+\infty, T(\tau) \rightarrow 0$.

Next, we will prove that for an arbitrary but fixed $L>0$, there exists a unique $\alpha=\alpha(c) \in(0,+\infty)$ such that $T(\alpha(c))=$ $L$ is a fundamental period of the cnoidal wave solution (47). So, we have the following theorem.

Theorem 2. Let $L>0$ but fixed. Consider $c_{0}>0$ and the unique $\tau_{0}=\tau_{0}\left(c_{0}\right) \in(0,+\infty)$ such that $T\left(\tau_{0}\right)=L$. Then, there exists an internal $A\left(c_{0}\right)$ around $c_{0}$, an internal $B\left(b_{0}\right)$ around $b_{0}$, and a unique smooth function $\Upsilon$ such that $\Upsilon\left(c_{0}\right)=\alpha_{0}$ and

$$
L=\frac{4 K(k)}{\tau}
$$

where $c \in A\left(c_{0}\right), \tau=\Upsilon(c)$.

Proof. The proof is similar to that of Theorem 1. For details see Theorem 1 (see also Angulo Pava $[6,8,9]$ ).

\section{The Periodic Solution for Zakharov-Rubenchik Equations}

Now, we consider the Zakharov-Rubenchik equations:

$$
\begin{aligned}
i B_{t}+\omega B_{x x}-\ell\left(u-\frac{1}{2} \lambda \rho+q|B|^{2} B\right) & =0, \\
\theta \rho_{t}+(u-\lambda \rho)_{x} & =-\ell|B|_{x}^{2}, \\
\theta u_{t}+(\beta \rho-\lambda u)_{x} & =\frac{1}{2} \ell \lambda|B|_{x}^{2},
\end{aligned}
$$


which describe the dynamics of small amplitude Alfvén waves propagating in a plasma. Here, $B(x, t)$ denotes the magnetic field, $u(x, t)$ the fluid speed, and $\rho(x, t)$ the density of mass. Moreover, $\omega, \ell, \lambda, \theta, \beta, q$ are real constants.

Motivated by Oliveira [10], we look for the solitary waves of (51), as follows:

$$
\begin{aligned}
B(x, t) & =e^{i \xi} A(\xi), \\
u(x, t) & =a A^{2}(\xi), \\
\rho(x, t) & =b A^{2}(\xi), \\
\xi & =x-c t,
\end{aligned}
$$

where $a, b$, and $A$ are real functions and $c$ denotes traveling speed.

Putting (52) into (51), from the second and third equations of (51), we can deduce

$$
\begin{aligned}
& a=a(c)=\frac{\ell[(\lambda / 2)(\lambda+c \theta)-\beta]}{\beta-(c \theta+\lambda)^{2}}, \\
& b=b(c)=\frac{\ell(-c \theta-\lambda / 2)}{\beta-(c \theta+\lambda)^{2}} .
\end{aligned}
$$
that

Moreover, from the first equation of (51), we can obtain

$$
\begin{array}{r}
(c-\omega) A+i(2 \omega-c) A^{\prime}+\omega A^{\prime \prime} \\
-\ell\left(a-\frac{1}{2} \lambda b+q\right) A^{3}=0 .
\end{array}
$$

So, by (54), it implies

$$
\begin{aligned}
c & =2 \omega, \\
A^{\prime \prime}+A-\frac{2 \ell(a-(\lambda / 2) b+q)}{c} A^{3} & =0 .
\end{aligned}
$$

Let $b_{1}=-\ell(a-(\lambda / 2) b+q) / c>0$ and multiplying (56) by $A^{\prime}$ and integrating once, we obtain

$$
\left[A^{\prime}\right]^{2}=b_{1}\left(A^{2}+a_{1}^{2}\right)\left(b_{2}^{2}-A^{2}\right)
$$

where $-a_{1} i, a_{1} i,-b_{2}, b_{2}$ are the polynomial roots of $F(r)=$ $-b_{1} r^{4}-r^{2}+h$. Moreover,

$$
\begin{gathered}
b_{2}^{2}-a_{1}^{2}=-\frac{1}{b_{1}}, \\
b_{2}^{2} a_{1}^{2}=\frac{h}{b_{1}} .
\end{gathered}
$$

Let $\chi=A / b_{2}$ (suppose $\left.\chi(0)=0\right)$ and $k^{2}=b_{2}^{2} /\left(a_{1}^{2}+b_{2}^{2}\right)$. Hence, (57) becomes

$$
\left(\chi^{\prime}\right)^{2}=b_{1} b_{2}^{2}\left(1-\chi^{2}\right)\left(\frac{a_{1}^{2}}{b_{2}^{2}}+\chi^{2}\right)
$$

Now, we define $\chi^{2}=1-\sin ^{2} \psi$, so we get that

$$
\left(\psi^{\prime}\right)^{2}=b_{1}\left(a_{1}^{2}+b_{2}^{2}\right)\left(1-k^{2} \sin ^{2} \psi\right)
$$

Let $\alpha=\sqrt{b_{1}\left(a_{1}^{2}+b_{2}^{2}\right)}$. According to the definition of the Jacobin elliptic function sn, we can obtain $\sin \psi(\xi)=$ $\operatorname{sn}(\alpha \xi, k)$. So, $\chi^{2}=1-\operatorname{sn}^{2}(\alpha \xi, k)$.

So, we obtain the solution of (56):

$$
A(\xi)=b_{2} \operatorname{cn}(\alpha \xi, k) \text {. }
$$

Since cn has fundamental period $4 K(k)$, we can obtain that solution (61) has fundamental period, $T$, which is

$$
T=\frac{4 K(k)}{\sqrt{b_{1}\left(a_{1}^{2}+b_{2}^{2}\right)}} .
$$

Hence, (51) have the periodic solutions of the following form:

$$
\begin{aligned}
& B(\xi)=e^{i \xi} b_{2} \mathrm{cn}(\alpha \xi, k), \\
& u(\xi)=a b_{2}^{2} \mathrm{cn}^{2}(\alpha \xi, k), \\
& \rho(\xi)=b b_{2}^{2} \mathrm{cn}^{2}(\alpha \xi, k) .
\end{aligned}
$$

Moreover, from (58) and the definition of $k^{2}$, it follows that

$$
k^{2}=\frac{a_{1}^{2}-1 / b_{1}}{2 a_{1}^{2}-1 / b_{1}},
$$

$$
T\left(a_{1}\right)=\frac{4 K(k)}{\sqrt{2 a_{1}^{2} b_{1}-1}} .
$$

Moreover, if $a_{1} \rightarrow 0, k^{2} \rightarrow 1, T\left(a_{1}\right) \rightarrow+\infty$. If $a_{1} \rightarrow+\infty$, $k^{2} \rightarrow 1 / 2, T\left(a_{1}\right) \rightarrow 0$.

Next, we will show that for an arbitrary but fixed $L>0$, there exists $a_{1}=a_{1}(c) \in(0,+\infty)$ such that $T\left(a_{1}(c)\right)=L$ is a fundamental period of the cnoidal wave solution (61). Motivated by Angulo Pava's result [6], we have the following theorem.

Theorem 3. For $L>0$ arbitrary and fixed, consider $c_{0}>0$ and the unique $a_{1}=a_{1}\left(c_{0}\right) \in(0,+\infty)$. Then, there exist an interval $C\left(c_{0}\right)$ around $c_{0}$, an interval $D\left(a_{1}\right)$ around $a_{1}$, and a unique smooth function $H: C\left(c_{0}\right) \rightarrow D\left(a_{1}\right)$ such that $H\left(c_{0}\right)=a_{1}$ and

$$
L=\frac{4 K(k)}{\sqrt{2 a_{1}^{2} b_{1}-1}},
$$

where $c \in C\left(c_{0}\right), a_{1}=H(c)$.

Proof. The idea and method are similar to those of Theorem 1. For details, please see Theorem 1 (see also Angulo Pava [6, 8, 9]). 


\section{Conclusion}

Inspired by Angulo Pava's idea, by applying Jacobian elliptic function method, we have obtained new period wave solution for Klein-Gordon Zakharov equations, Zakharov equations, and Zakharov-Rubenchik equations. In particular, the solutions of (18), (47), and (58) were not found in the previous work. The method can help to look for periodic solution for a class of nonlinear equations.

\section{Competing Interests}

The authors declare that there is no conflict of interests regarding the publication of this article.

\section{Acknowledgments}

This work was supported by Scientific Research Foundation of the City College of Jilin Jianzhu University (no. 2016002), NSFC Grant (nos. 11322105, J1310022, and 11671071) and 973 Program (nos. 2012CB821200 and 2013CB834102).

\section{References}

[1] M. L. Wang, "Solitary wave solutions for variant Boussinesq equations," Physics Letters A, vol. 199, no. 3-4, pp. 169-172, 1995.

[2] C. T. Yan, "A simple transformation for nonlinear waves," Physics Letters A, vol. 224, no. 1-2, pp. 77-84, 1996.

[3] S. K. Liu, Z. T. Fu, S. D. Liu, and Q. Zhao, "A simple fast method in finding particular solutions of some nonlinear PDE," Applied Mathematics and Mechanics, vol. 22, no. 3, pp. 326-331, 2001.

[4] E. J. Parkes and B. R. Duffy, "Travelling solitary wave solutions to a compound KdV-Burgers equation," Physics Letters A, vol. 229, no. 4, pp. 217-220, 1997.

[5] E. Fan, "Extended tanh-function method and its applications to nonlinear equations," Physics Letters A, vol. 277, no. 4-5, pp. 212-218, 2000.

[6] J. Angulo Pava, "Nonlinear stability of periodic traveling wave solutions to the Schrödinger and the modified Korteweg-de Vries equations," Journal of Differential Equations, vol. 235, no. 1, pp. 1-30, 2007.

[7] P. F. Byrd and M. D. Friedman, Handbook of Elliptic Integrals for Engineers and Scientists, Springer, New York, NY, USA, 2nd edition, 1971.

[8] J. Angulo Pava, "Stability of cnoidal waves to Hirota-Satsuma systems," Matemática Contemporânea, vol. 27, pp. 189-223, 2004.

[9] J. Angulo Pava, Nonlinear Dispersive Equations: Existence and Stability of Solitary and Periodic Travelling Wave Solutions, vol. 156 of Mathematical Surveys and Monographs, American Mathematical Society, Providence, RI, USA, 2009.

[10] F. Oliveira, "Stability of the solitons for the one-dimensional Zakharov-Rubenchik equation," Physica D, vol. 175, no. 3-4, pp. 220-240, 2003. 


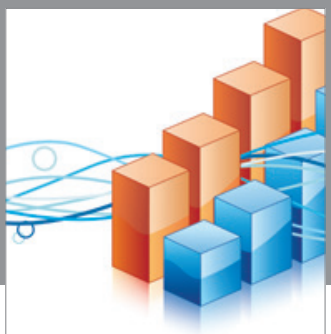

Advances in

Operations Research

vatem alat4

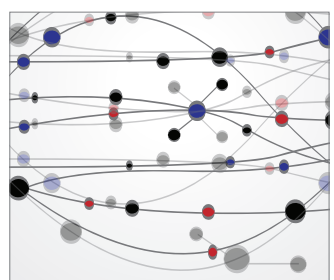

\section{The Scientific} World Journal
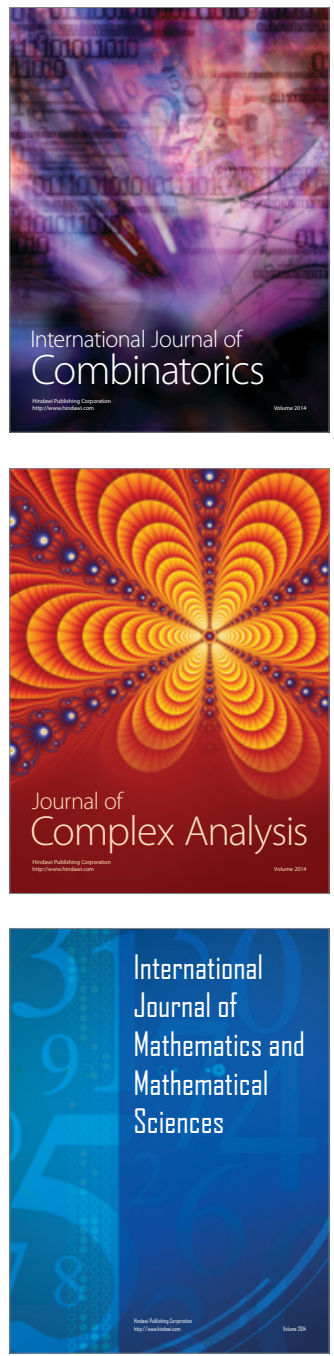
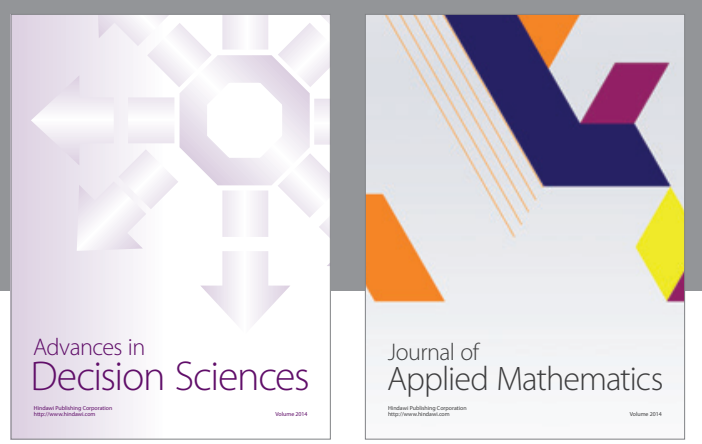

Algebra

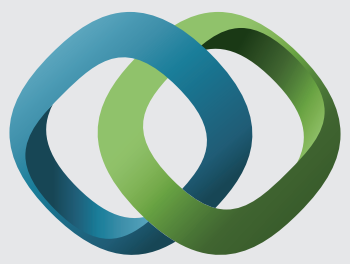

\section{Hindawi}

Submit your manuscripts at

http://www.hindawi.com
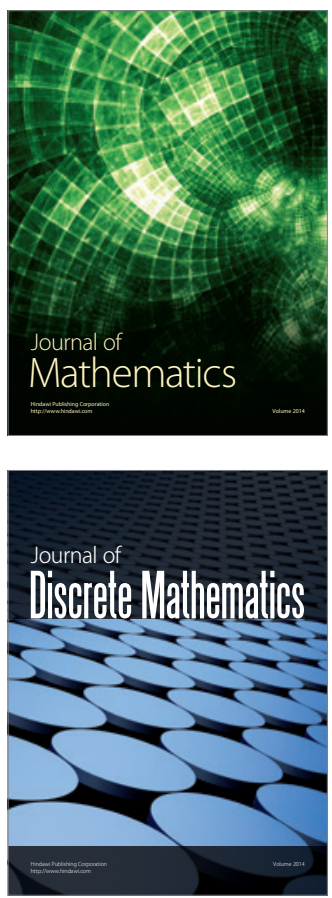

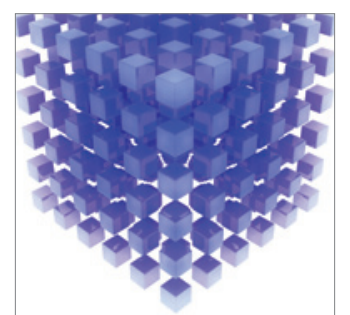

Mathematical Problems in Engineering
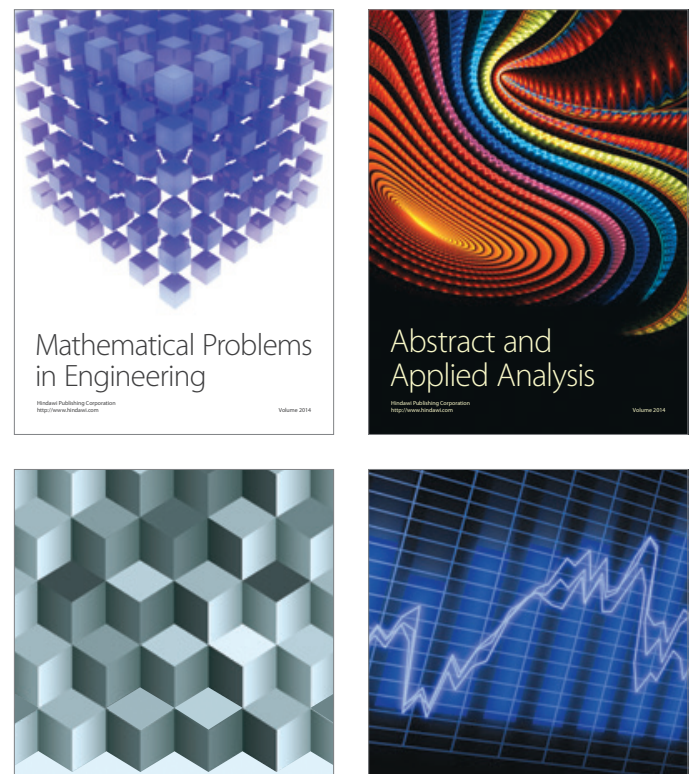

Journal of

Function Spaces

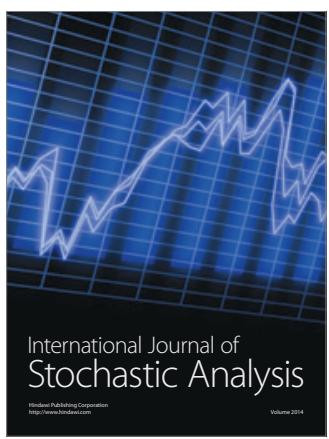

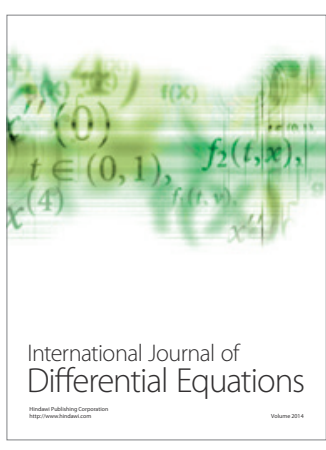
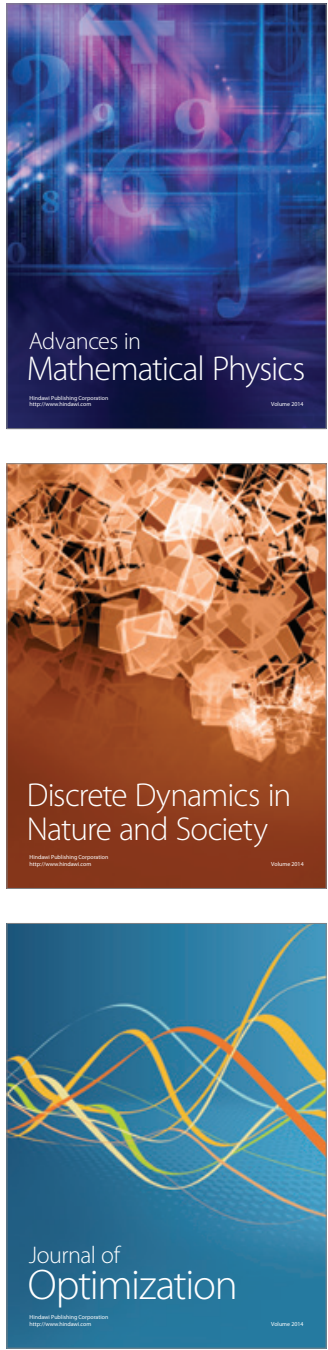